\title{
DETERMINATION OF THE TIME SINCE DEATH BY HISTOLOGICAL CHANGES IN DISTAL CONVOLUTED TUBULE IN HUMAN KIDNEYS
}

\author{
Rajni Thakur *1, Anjana Tiwari ${ }^{2}$. \\ ${ }^{{ }_{1} 1}$ Assistant Professor, Department of Anatomy, Pt.J.N.M.Medical College, Raipur, (C.G.), India. \\ ${ }^{2}$ Associate Professor, Department of P.S.M, Chandulal Chandrakar Medical College, Durg, (C.G.), \\ India.
}

\section{ABSTRACT}

\begin{abstract}
Background: The DCT is lined with simple cuboidal cells that are shorter than those of the proximal convoluted tubule (PCT). The lumen appears larger in DCT, the histological changes DCT in kidney after death have been studied in various land animals and sea marine animals but yet very few studies with same view which may provide keen and fruitful results for human distal convoluted tubule in kidney have been done. It will be helpful for estimation of time after death.
\end{abstract}

Aim: To determine the time since death by histological changes in Distal convoluted tubule.

Materials and Methods: 50 Human kidneys samples at different time intervals between $4 \mathrm{hrs}$ to $52.30 \mathrm{hr}$, temperature between $17.3 / 22.3-31.3 / 45^{\circ} \mathrm{C}$, humidity between $11 / 36$ to $75 / 95$, were of different age and sex obtained as and when available from cadavers with a known time of death where death had resulted from natural death, suffocation and trauma, dead individual health and not suffering from disease affecting kidney.

Result: Increase the rate of postmortem histological changes in DCT was found to be increased with rise in the temperature and duration. In Distal convoluted tubules lumens were found swelling of epithelial cells. Retraction of epithelium from the basement membrane and its disruption with individualization of cells, nuclear pyknosis, karyolysis and loss of tubular architecture with debris in the lumen were observed.

Conclusion: In this current research, sequence of postmortem histological changes in DCT was studied. These criteria's presented in this study could be used to determine the time after death.

KEY WORDS: Retraction, Debris, Duration, DCT, Pyknosis, Humidity.

Corresponding Author: Dr. Rajni Thakur, Assistant Professor, Department of Anatomy, Pt.J.N.M.Medical College, Raipur, (C.G.), India. Mobile No.: 9424203190.

E-Mail: dr.tistathakur2004@gmail.com.

Access this Article online

Quick Response code

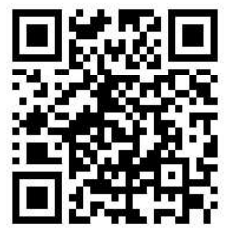

DOI: $10.16965 /$ ijar.2019.310

Journal Information

\section{International Journal of Anatomy and Research}

ICV for 2016 ISSN (E) 2321-4287 | ISSN (P) 2321-8967

90.30 https://www.ijmhr.org/ijar.htm

DOI-Prefix: https://dx.doi.org/10.16965/ijar

(c) $\mathrm{B}+\mathrm{Mi}-\mathrm{Sh}$

Article Information

Received: 03 Sep 2019

Peer Review: 03 Sep 2019

Revised: None
Accepted: 09 Oct 2019

Published (O): 05 Nov 2019

Published (P): 05 Nov 2019

\section{INTRODUCTION}

The distal convoluted tubule (DCT) is a portion of kidney nephron between the loop of Henle and the collecting tubule. The DCT is lined with simple cuboidal cells that are shorter than those of the proximal convoluted tubule (PCT). The lumen appears larger in DCT than the PCT lumen because the PCT has a brush border (microvilli). DCT can be recognized by its numerous mitochondria, basal enfolding and lateral membrane interdigitations with neighboring cells [1]. After death, a sequence of changes naturally occurs in the human body. Although these changes proceed in a relatively orderly fashion, a variety of external factors and intrinsic characteristics may accelerate or retard 
decomposition. Understanding common postmortem changes and the variables that affect them allows the forensic pathologist to more accurately estimate the postmortem interval (PMI) and to provide a time frame during which death occurred. Further, an awareness of common postmortem artifacts limits the risk of misdiagnosis at the time of autopsy [2].

The time of death is sometimes extremely important. It is a question almost invariably asked sometimes with a touching faith in the accuracy of the estimate. Determining the time of death is extremely difficult, and accuracy is impossible. In this study control cannot be taken because the histological changes of tissue after death is influenced a great deal by atmospheric temperature and humidity besides other external and internal factors [3]. Criteria for autolysis comprised the following factors. The overall severity of autolysis was based on the number of factors present and the magnitude of change in the affected cells: pyknosis, karyorrhexis, karyolysis, the absence of a nucleus due to complete dissolution or lysis, cellular edema/swelling, failure to take up stain, intracytoplasmic vacuolation, putrefaction, altered architecture of tissue unrelated to a pathological process [4]. Gradual decrease of body temperature is one of the earliest sign of death. The formula for estimation of postmortem interval from rectal temperature and abdominal temperature [5].

In past, the histological changes DCT in kidney after death have been studied in various land animals and sea marine animals but yet very few studies with same view which may provide keen and fruitful results for human distal convoluted tubule in kidney have been done. That's why this current study is being carried out with this hope that it will be helpful for estimation of time after death which is very critical and one of the most important job for a forensic, anatomist and upcoming scientist.

\section{MATERIALS AND METHODS}

This present research was done in Department of Anatomy in close association with the Department of Forensic Medicine \& Toxicology and Pathology, Pt.J.N.M.Medical College, Raipur (C.G.). 50 Human kidneys samples were of different age and sex obtained as and when available from cadavers at the time of autopsy. It was removed from cadavers with a known time of death where death had resulted from natural death, suffocation and trauma, dead individual health and not suffering from disease affecting kidney. Cases exclude dead individual those preserve in ice or ice cooler, unknown time of death, suffering from kidney disease, cases complicated by other metabolic disorders like Diabetic mellitus, renal oesteodystrophy, secondary hyperparathyroidism nutritional disturbance (PEM).The stages for which it was available temperature between17.3/22.3-31.3/ $45^{\circ} \mathrm{C}$, the environmental temperature in $\mathrm{p} \mathrm{C}$ [minimum/maximum] and humidity in \% [minimum/maximum] was recorded from "India Meteorological Department, Meteorological Centre Raipur". Humidity between $11 / 36$ to $75 / 95$ and duration range was between $4 \mathrm{hrs}$ to $52.30 \mathrm{hr}$. In the current research, each case was studied by histological (H\& E staining, PAS staining).

\section{OBSERVATION AND RESULTS}

Postmortem 50 Human kidneys samples at different time intervals between $4 \mathrm{hrs}$ to $52.30 \mathrm{hr}$. , In this research increase the rate of postmortem histological changes in DCT were found to be increased with rise in the temperature and duration. There were significant changes observed. We divided into 4 groups.

Group: I - In this group included post mortem interval (PMI) - $4 \mathrm{hrs}$ Temperature-27.5/42. ${ }^{\circ} \mathrm{C}$, humidity-14/41\% to PMI $13 \mathrm{hrs}$ Temp- $23.9 / 38.7^{\circ} \mathrm{C}$ humidity-41/72\%, Distal convoluted tubule was show following progressive degenerative changes - disruption of epithelium at most of places with dark stained nuclei, then retraction of epithelium from the basement membrane, throughout while it is disrupted at places, vesicular and dark stained nuclei, at places edematous cells are also present. There after DCT expanded disruption of epithelium most of the places enucleated epithelial, cells, debris in the lumen with mostly dark stained nuclei. (Fig. 1, Fig. 2, Fig. 3).

Group: II - In this group included PMI 13hrs Temp- $25.5 / 31^{\circ} \mathrm{C}$ humidity-85/87\% TO PMI $17 \mathrm{hrs}$ Temp- $12.8 / 23.5^{\circ} \mathrm{C}$ humidity- $44 / 82 \%$, Distal 

HUMAN KIDNEYS.

convoluted tubule was show following degenerative changes - retraction and disruption of epithelium, enucleated epithelial cells with vesicular and dark nuclei almost present. (Fig. 4, Fig. 5).

Group: III - In this group included PMI 17.30hrs Temp- $27.3 / 42.2^{\circ} \mathrm{C}$ humidity-24/52\% to PMI $21 \mathrm{hrs}$ Temp- $25.5 / 31^{\circ} \mathrm{C}$ humidity- $85 / 87 \%$, following degenerative changes was show, in Distal convoluted tubule - disruption of epithelium with dark stained as well as vesicular nuclei and debris present in the lumen, but enucleated also at few places, epithelium is not retracted from the basement membrane at some places. (Fig.6).

Group: IV - In this group included PMI $21 \mathrm{hrs}$ Temp- $25.5 / 31^{\circ} \mathrm{C}$ humidity- $85 / 87 \%$ to $\mathrm{PMI}$ $52.30 \mathrm{hrs}$ Temp- $24.5 / 32^{\circ} \mathrm{C}$ humidity-75/95\%, following degenerative changes was show, in Distal convoluted tubule - retraction and disruption of epithelium with dark stained nuclei, Enucleated epithelial cells and individualization of the cells are seen at places, then dilated, retraction and disruption of epithelium, after then dilated with wide lumen, debris present in the lumen, disruption of epithelium with nuclei dark stained at their peripheral part, pyknotic changes are hardly seen, after then very fast changes in DCT - retraction and disruption of epithelium, cellular debris in the lumen with pyknotic nuclei. At 52.30hrs show DCT- are not identifiable in most of the places, disruption of epithelium at places with pyknotic nuclei but anucleated epithelial cells and individualization of cells are also present and debris present in the lumen. (Fig. 7, Fig. 8, Fig. 9).

Fig. 1. (A) \& (B): 4 hrTemp. $42.2 / 27.5^{\circ} \mathrm{C}$ H\&E stains $10 \mathrm{X}$. Photomicrograph showing in DCT- disruption of epithelium at most of places with dark stained nuclei.DCT was PAS+.

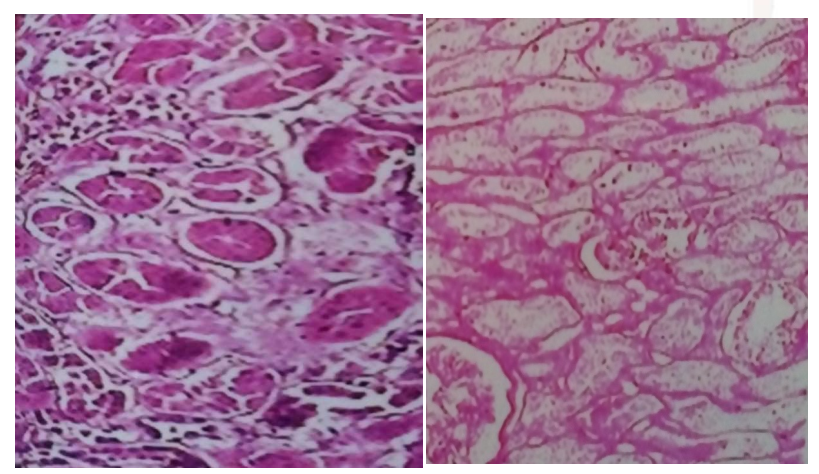

Fig. 2: $5 \mathrm{hr}$ Temp. $18.1 / 34.6^{\circ} \mathrm{C}$ H\&E stains high power, Photomicrograph showing in DCT- retraction of epithelium from the basement membrane however the structure of the epithelium is maintained with dark stained nuclei was seen.

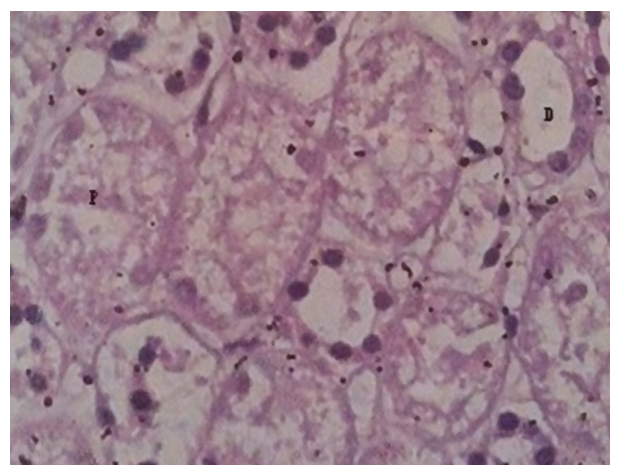

Fig. 3: $13 \mathrm{hr}$ Temp. $23.9 / 38.7^{\circ} \mathrm{C}$ H\&E stains 10X. Photomicrograph showing in DCT- Disruption of epithelium and debris in the lumen.

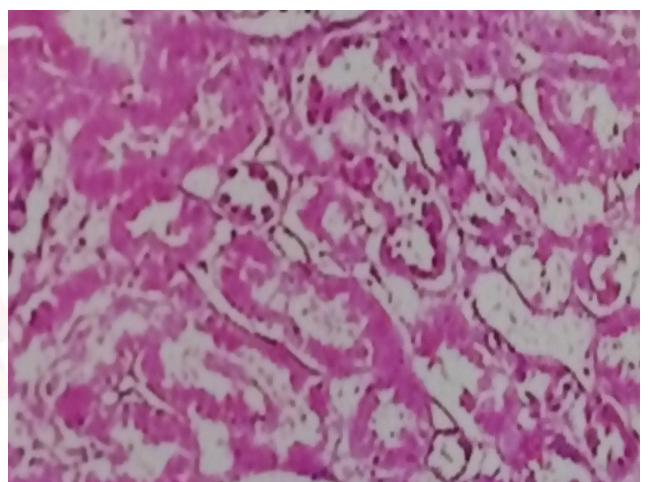

Fig. 4: $16 \mathrm{hr}$ Temp.27.5/42.20 C H\&E stains 10X. Photomicrograph showing in DCT- Disruption of epithelium.

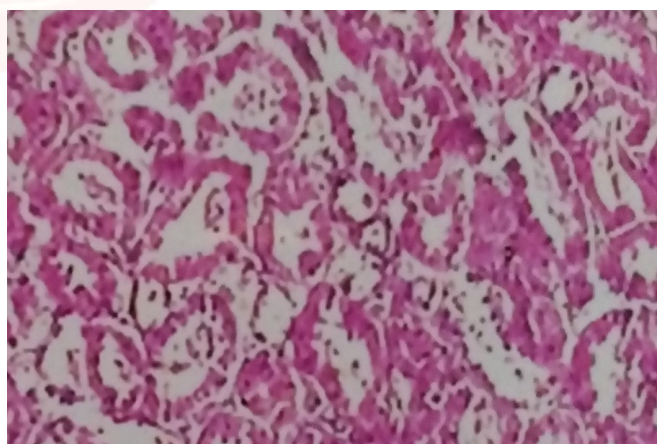

Fig. 5: $16.30 \mathrm{hr}$ Temp. $21 / 39^{\circ} \mathrm{C}$ H\&E stains high power Photomicrograph showing in DCT- retraction and disruption of epithelium with dark stained nuclei mostly, vesicular nuclei also seen.

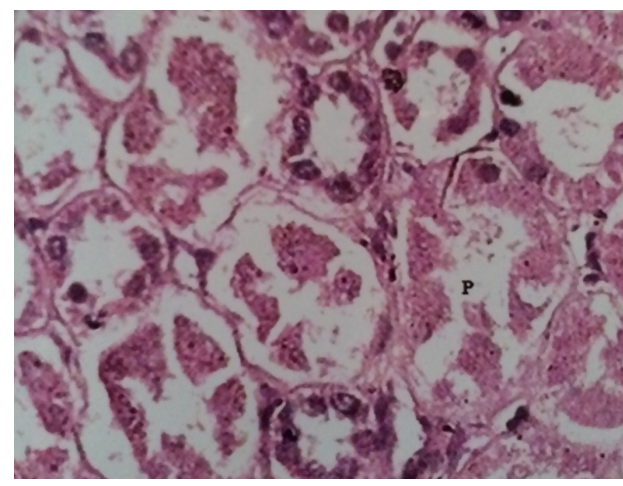


Fig. 6: $20 \mathrm{hr}$ Temp. $25.5 / 31^{\circ} \mathrm{C}$ H\&E stains high power Photomicrograph showing in DCT- retraction epithelium with disruption at places having dark stained nuclei. Individualization of epithelial cells. In lumen of the tubules debris present.

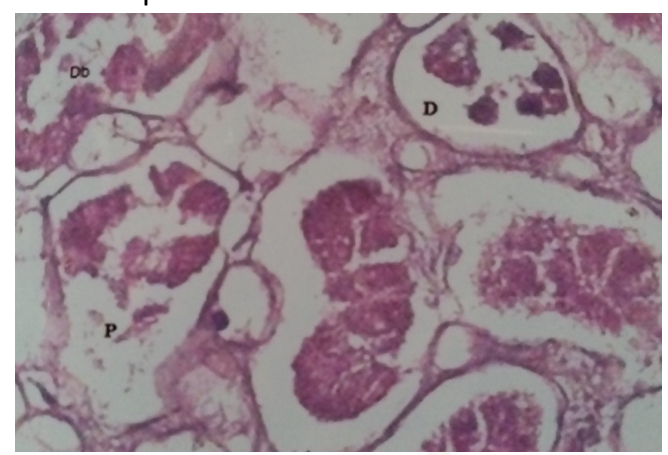

Fig. 7: $21.30 \mathrm{hr}$ Temp. $26 / 40.8^{\circ} \mathrm{C}$ H\&E stains $10 \mathrm{X}$. Photomicrograph showing in DCT- retraction, disruption of epithelium, debris present in the lumen, individualization of cells at places.

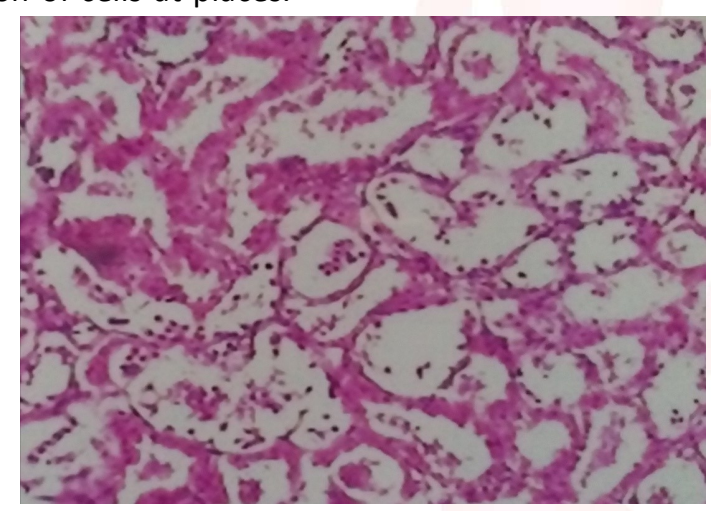

\section{DISCUSSION}

In the current research remarkable postmortem histological changes were seen in DCT, sequence of histological changes in DCT were studied, retraction as well as disruption (fragmented) of epithelium from the basement membrane although the cells were with nuclei, PAS+, Forensic medicine practice shows continuous expert, scientific, ethical, and legal need to, besides identifying the cause of death, we identify the time of death as precise as possible. The most common method for establishing the postmortem time is based on the development of corpse's characteristics and changes which depend on variable factors such as the temperature, air moisture and the surroundings [6,7]. The main reason lies in the fact that there are an extreme number of factors, which influence the post-mortal degradation of tissue in each concrete case [8]. This problem had been seen as a forensic one for a long time, but later, especially in the second half of the last century it became more important due to the use of new
Fig. 8: PAS staining PMI 28hrs Temp-26.7 $\beta 8.7^{\circ} \mathrm{C}$, DCTwas partly PAS +.

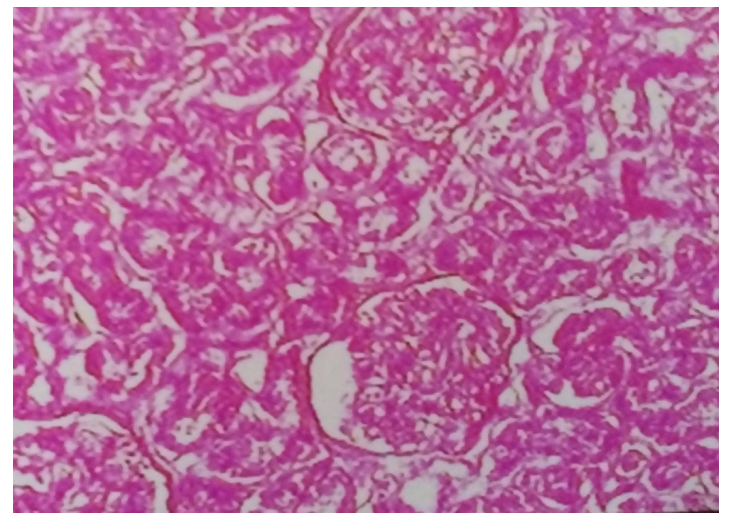

Fig. 9: 52.30hr Temp.24.5/32.0 C H\&E stains 10X. Photomicrograph showing in DCT- disruption of epithelium of epithelium with pyknotic nuclei but enucleated epithelial cells and individualization of cells are also present.

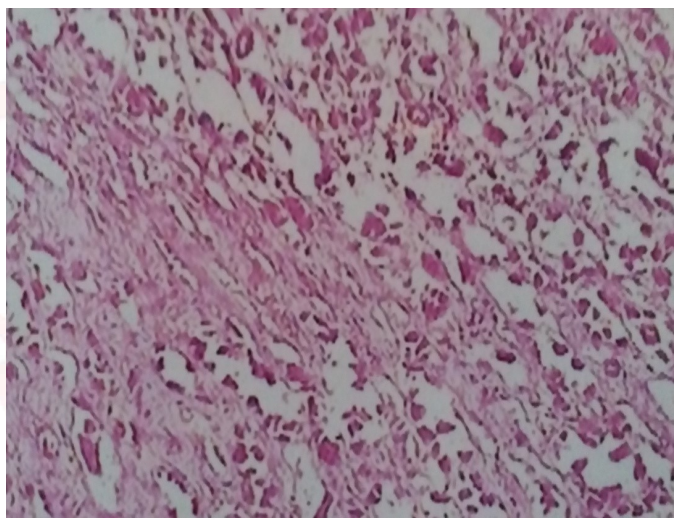

treatment methods in modern medicine referring to the tissue and organ transplantations. That's why the determination of the time of death problem in the current level of medical science and technique became more popular, not only for the forensics, but also for some other biomedical specialties: surgeons, nephrologists, traumatologists, hematologists, transplatators, etc [9].

In the current research, group: I, post mortem interval (PMI) - $4 \mathrm{hrs}$ Temperature-27.5/42.2 ${ }^{\circ} \mathrm{C}$, humidity-14/41\% to PMI $13 \mathrm{hrs}$ Temp- $23.9 / 38.7^{\circ} \mathrm{C}$ humidity-41/72\%, Distal convoluted tubule was show following progressive degenerative changes - disruption of epithelium at most of places with dark stained nuclei, then retraction of epithelium from the basement membrane, throughout while it is disrupted at places, vesicular and dark stained nuclei, at places oedematous cells are also present. There after DCT expanded disruption of epithelium most of the places enucleated epithelial, cells, debris in the lumen with mostly dark stained nuclei. Some author like Tomita et al [10], found in wistar 
rats, 5 hours after death, PM changes were clumping of nuclear chromatin in proximal tubules as well as distal tubules. Deborah Barber [11] found in Porcine kidney, 6 Hours $24^{\circ} \mathrm{C}$, DCT-Most of the cells have sloughed off the basement membrane and formed cellular debris within the lumen. Few cells remain in contact with the basement membranes. Rakesh Tadon [12] found at $30^{\circ} \mathrm{C}$ cloudy swelling of DCT was seen by $12 \mathrm{hrs}$ after death. Samar Omar Rabah [13] found in $12 \mathrm{hrs}$, degenerative changes and marked atrophy of distal tubules cells.

In the current research, Group: II - In this group included PMI $13 \mathrm{hrs}$ Temp- $25.5 / 31^{\circ} \mathrm{C}$ humidity$85 / 87 \%$ TO PMI $17 \mathrm{hrs}$ Temp- $12.8 / 23.5^{\circ} \mathrm{C}$ humidity-44/82\%, Distal convoluted tubule was show following degenerative changes - retraction and disruption of epithelium, enucleated epithelial cells with vesicular and dark nuclei almost present. Some author like, Vinita kushwaha et al [14] found in their study after 13-18 hrs PMI, with increasing temperature of up to 31 to $35^{\circ} \mathrm{C}$, found moderate\& severe changes are seen. Architecture maintained, more cloudy swelling and disruption of epithelium, Tomita et $\mathrm{al}^{10}$ found at 15 hours after death cytolysis of distal tubules.

In the current research, group: III - In this group included PMI $17.30 \mathrm{hrs}$ Temp- $27.3 / 42.2^{\circ} \mathrm{C}$ humidity-24/52\% to PMI $21 \mathrm{hrs} \mathrm{Temp-} 25.5 / 31^{\circ} \mathrm{C}$ humidity-85/87\%, in Distal convoluted tubule was show following degenerative changes, disruption of epithelium with dark stained as well as vesicular nuclei and debris present in the lumen, but enucleated also at few places, epithelium is not retracted from the basement membrane at some places and group: IV, PMI $21 \mathrm{hrs}$ Temp- 25.5/ $31^{\circ} \mathrm{C}$ humidity- $85 / 87 \%$ to PMI $52.30 \mathrm{hrs}$ Temp$24.5 / 32^{\circ} \mathrm{C}$ humidity-75/95\%, in Distal convoluted tubule was show retraction and disruption of epithelium with dark stained nuclei, Enucleated epithelial cells and individualization of the cells are seen at places, then dilated, retraction and disruption of epithelium, after then dilated with wide lumen, debris present in the lumen, disruption of epithelium with nuclei dark stained at their peripheral part, pyknotic changes are hardly seen, after then very fast changes in DCT - retraction and disruption of epithelium, cellular debris in the lumen with pyknotic nuclei. At $52.30 \mathrm{hrs}$ show DCT- are not identifiable in most of the places, disruption of epithelium at places with pyknotic nuclei but enucleated epithelial cells and individualization of cells are also present and debris present in the lumen, PAS+ substances were not seen. Some author like, Doha Yahia at el [15] found in dog's kidney, liver, heart, and skeletal muscles within $72 \mathrm{~h}$ after death. Histopathological changes in the kidneys were the earliest changes seen in tissue and can be used for estimating the postmortem intervals in dogs. Yukari Tomita at et [16], found most of the ultra structural changes occurred earlier in kidney. In hepatocytes, the morphological degeneration occurred later than in the renal tubule epithelium. A. Mamun at el [17], found swelling appearances, highly degeneration and edema of renal tubules. Miodrag Zdravkoviæ at et ${ }^{18}$ found 112 experimental rats a PAS histochemical analysis of renal cortex specimens, from 1 to $72 \mathrm{~h}$. tubular basal membrane and apical parts of tubular epithelial cells. The dynamic of the appearance of morphological post mortal autolytic changes depends on the time of death and on the temperature at which the autolysis is evolving and also, that there is a certain autolytical order depending on the environmental conditions.

During last decades many postmortem interval studies referring to the establishing of biochemical, histological, histochemical and ultra structural changes in different tissues and organs were done $[19,20]$. This current researches could be used to determine the time after death.

\section{CONCLUSION}

In the current research sequence of postmortem histological changes in DCT were studied. In Distal convoluted tubules lumens were found swelling of epithelial cells. Retraction of epithelium from the basement membrane and its disruption with individualization of cells, nuclear pyknosis, karyolysis and loss of tubular architecture with debris in the lumen were observed.

\section{ACKNOWLEDGEMENTS}

The author was grateful to her adorable parents late Shree D.R.Mandavi, late Smt. Durga 

HUMAN KIDNEYS.

Mandavi and lovable brother late Shree Rajesh Mandavi for their blessings and inspirations. The authors are highly thankful to head of all three departments and their staff of Pt.J.N.M.Medical college Raipur, Chhattisgarh, India for their cooperation and support during the entire study period and also thankful to authors/ editors/publisher of all those articles journals which are cited and included in references of this manuscript.

\section{Conflicts of Interests: None}

\section{REFERENCES}

[1]. https://en.wikipedia.org/wiki/Distal convoluted tubule 26 February 2019, at 07:58 (UTC).

[2]. S.Erin Presnell ; Chief Editor ,et al, Postmortem Changges:Oct 13, 2015

[3]. Knight (1987): legal Aspect of medical practice $4^{\text {th }}$ edition.

[4]. Jami George at el. Histopathologic evaluation of postmortem autolytic changes in bluegill (Lepomis macrohirus) and crappie (Pomoxis anularis) at varied time intervals and storage temperatures Pub Med 27114885, April 19, 2016.

[5]. Marshal and Hoare (1962): The rectal cooling after death its mathematical expression, J, Forensic Sci., 1962;7:56.

[6]. Knight B. Forensic Pathology. 2nd ed. Arnold, London, 1997.

[7]. Janssen W. Forensic histopathology. Springer Verlag, Berlin. 1985.

[8]. Micozz MS et al. Moisture inhibite the decomposition process of tissue Forensic Res 3:P;176.

[9]. Opelz G. Dohler B. Multicenter analysis of kidney preservation. Transplantation 2007;83(3):247-57.

[10].Tomita et al. Nihan Hoigaku zasshi, Baltimore mariland, 1993;53(2)2007-17.
[11]. Deborah Barber. Sequential Histologic postmortem changes in porcine kidney and Adrenal glands, Department of Pathology, D.V.M.Kansas state University. 1980.

[12]. Rakesh Tandon. Time and Temperature controlled histopathological changes in tissue \& organs of Rabbit. I.J.F. Sci P 1985;20-25.

[13]. Coe J. Postmortem chemistry update, emphasis on forensic application. Is J For Med Pathol 1993;14:91117.

[14]. Samar Omar Rabah. Acute taxol neprotoxicity: Histological and ultrastructural studies of mice kidney parenchyma,Sardi Journal of biological Science 2010;17:105-114.

[15]. Vinita Kushwaha et al. Time since death from degenerative changes in the kidney, J.Indian Academy of Forensic medicine 2010;32((1):37.

[16]. Doha Yahia at el, early postmortem biochemical and histopathological changes in the kidney, liver, and muscles of dogs. Comparative Clinical Pathology 2018;27(11):1447-1455.

[17]. Tomita Y, Nihira M, Ohno Y, Sato S. Ultrastructural changes during in situ early postmortem autolysis in kidney, pancreas, liver, heart and skeletal muscle of rats. Legal medicine. 2004 Mar 1;6(1):25-31.

[18]. A. Mamun at el, Histological study of the effect of Malathion on liver and kidney tissues of mice model, IJPSR. 2015; 6(3).1043-48,

[19]. Zdravkoviæ M, Kostov M, Stojanoviæ M. Identification of postmortem autolytic changes on the kidney tissue using PAS stained method. Med Biol. 2006;13(3):181-4.

[20]. Penttila A. Laiho K. Autolitic changes in blood of human cadavers. II morphological Studies. Forensic Sci Int 1981; 17(2): 121-132.

How to cite this article:

Rajni Thakur, Anjana Tiwari. DETERMINATION OF THE TIME SINCE DEATH BY HISTOLOGICAL CHANGES IN DISTAL CONVOLUTED TUBULE IN HUMAN KIDNEYS. Int J Anat Res 2019;7(4.2):7086-7091. DOI: 10.16965/ijar.2019.310 